\title{
Milky Way Rotation Models from Neutral Hydrogen and Molecular Clouds: Galactic Constants, Common Details and Differences
}

\author{
Igor' I. Nikiforov \\ Sobolev Astronomical Institute, St. Petersburg University, \\ Bibliotechnaya pl. 2, 198904 St. Petersburg, Russia
}

\section{Introduction}

Kinematic data from neutral hydrogen observations provide information to solve the interdependent problems of the determination of the main Galactic constants (the Solar-Galactic center distance $R_{0}$, the Oort constant $A$ and others) and the Galactic rotation curve (Nikiforov \& Petrovskaya 1994, hereafter NP94, and references therein). However, in the standard method for finding $R_{0}$ by comparing the rotations of $\mathrm{HI}$ clouds and some other objects (typically $\mathrm{HII}$ regions/CO clouds), the kinematic model, constructed typically solely from $\mathrm{H} \mathrm{I}$ data, is considered to be the same for both galactic subsystems (e.g. Merrifield 1992). In practice a discrepancy between their rotation curves can produce strongly erroneous results (Merrifield 1992, NP94). Establishing the common rotation law from $\mathrm{H}$ I plus $\mathrm{HII} / \mathrm{CO}$ data in NP94 is only a part of attacking the problem.

In this paper, techniques for the evaluation of $R_{0}$ which account for most of the effect of a difference between the rotation laws of $\mathrm{CO}$ clouds and $\mathrm{HI}$ is suggested. We derive the main Galactic constants, construct the rotation models, and discuss the difference between $\mathrm{HI}$ and $\mathrm{CO}$ rotations.

\section{Data}

For this study the $\mathrm{HI}$ tangent points and five independent $\mathrm{H}$ I data sets from the whole 21-cm line profile (see NP94) were taken. These latter contain Camm's function values $\Omega \equiv R_{0}\left(\omega-\omega_{\mathrm{LSR}}\right)$ in relation to $x \equiv R / R_{0}$, where $R$ is the distance to the galactic axis, $\omega$ and $\omega_{\mathrm{LSR}}$ are the angular velocities of $\mathrm{H} \mathrm{I}$ at the distance $R$ and at the Local Standard of Rest, respectively.

Two catalogues of CO clouds (COCs) from Brand \& Blitz's (1993) list were used here. In the first one ("BFS2") CO radial velocities relative to the LSR $V_{r}$, heliocentric distances $r$, and galactic coordinates $l, b$ were collected for 107 COCs associated with $\mathrm{H}$ II regions. For addition of data on southern COCs associated with $\mathrm{H}$ II regions or reflection nebulae (BBW sample), the second "BFS2/BBW-catalogue" (in all 209 objects) covers a greater longitude range, but it is less uniform in the object type. For more details, see Nikiforov (1999b). 


\section{Method}

Two fitting procedures are used in this study, both consisting of two steps.

The first step, refered to as "W(CO+HI) fitting", is the same for both procedures. It presents the way of using the $\mathrm{H}$ I and $\mathrm{CO}$ data to find the rotation model, common to these gaseous subsystems, with a given $R_{0}$.

Let us consider the function $W(x) \equiv x \Omega(x)=R\left[\omega(x)-\omega_{\mathrm{LSR}}\right]$, for which values can be calculated for every $\mathrm{H}$ I and $\mathrm{CO}$ data point. The following polynomial was selected as a model for the function $W(x)$ :

$$
W_{n}(x)=\sum_{i=0}^{n} w_{i}(x-1)^{i} .
$$

The obvious relations hold for coefficients in (1):

$$
w_{0}=-\Delta \theta_{\mathrm{LSR}}, \quad w_{1}=-2 A R_{0}-\Delta \theta_{\mathrm{LSR}},
$$

where $\Delta \theta_{\mathrm{LSR}}=\theta_{\mathrm{LSR}}-\theta_{0}, \theta_{\mathrm{LSR}}$ and $\theta_{0}$ are the linear rotation velocities of the LSR and gaseous subsystems (average for $\mathrm{H} I$ and $\mathrm{CO}$ ) at $R_{0}$, respectively. Using (2) the condition equations for observed values of $W(x)$ can be written

$$
W(x)=-\Delta \theta_{\mathrm{LSR}} x-2 A R_{0}(x-1)+\sum_{i=2}^{n} w_{i}(x-1)^{i} .
$$

For given $n$ and $R_{0}$ the system (3) is solved for $\Delta \theta_{\mathrm{LSR}}, A R_{0}$, and $w_{i}, i=2, n$. The best-fit solution is the one that minimizes the sum $\sum_{j} p_{j}\left[W(x)-W_{n}(x)\right]_{j}^{2}$ over all $\mathrm{HI}$ and $\mathrm{CO}$ data points. Values of $x$ and $\Omega$ for COCs and of weights $p_{j}$ were calculated in much the same way as NP94, but with some parameters revised and with the overall weight of $\mathrm{CO}$ data equated to the weight of $\mathrm{H} \mathrm{I}$ data (see Nikiforov 1999c).

Second step. To determine $R_{0}$ correctly one should take account of a possible difference between the rotation laws of $\mathrm{HI}$ and $\mathrm{CO}$. If we assume a constant differencial asymmetric drift between $\mathrm{H}$ I and $\mathrm{CO}$ subsystems we may consider the model parameters $A R_{0}$ and $w_{i}, i=2, n$, found at the first step, as known functions of $R_{0}$. Two variants of the second step are suggested here:

(i) $W(\mathrm{CO})$ fitting. The system of equations (3) can be solved for only $R_{0}$ and $\Delta \theta_{\mathrm{LSR}}$ from the CO data alone.

(ii) $W / V_{r}$ fitting. Under assumption of purely circular motions, the radial velocity field of COCs can be described by the following equation:

$$
\begin{aligned}
V_{r}= & {\left[-2 A R_{0}(x-1)+\sum_{i=2}^{n} w_{i}(x-1)^{i}\right] x^{-1} \sin l \cos b-} \\
& -\Delta \theta_{\mathrm{LSR}} \sin l \cos b-\Pi_{\mathrm{LSR}} \cos l \cos b .
\end{aligned}
$$

System (4) is solved for $R_{0}, \Delta \theta_{\mathrm{LSR}}$, and $\Pi_{\mathrm{LSR}}$ (the LSR radial motion in the direction $l=0^{\circ}$ ) by minimizing the sum of residual velocities squared. A difference between the $\Delta \theta_{\mathrm{LSR}}$ values obtained at the second and first steps represents a mean drift between the rotation curve for $\mathrm{COCs}$ and the average one over both subsystems. 


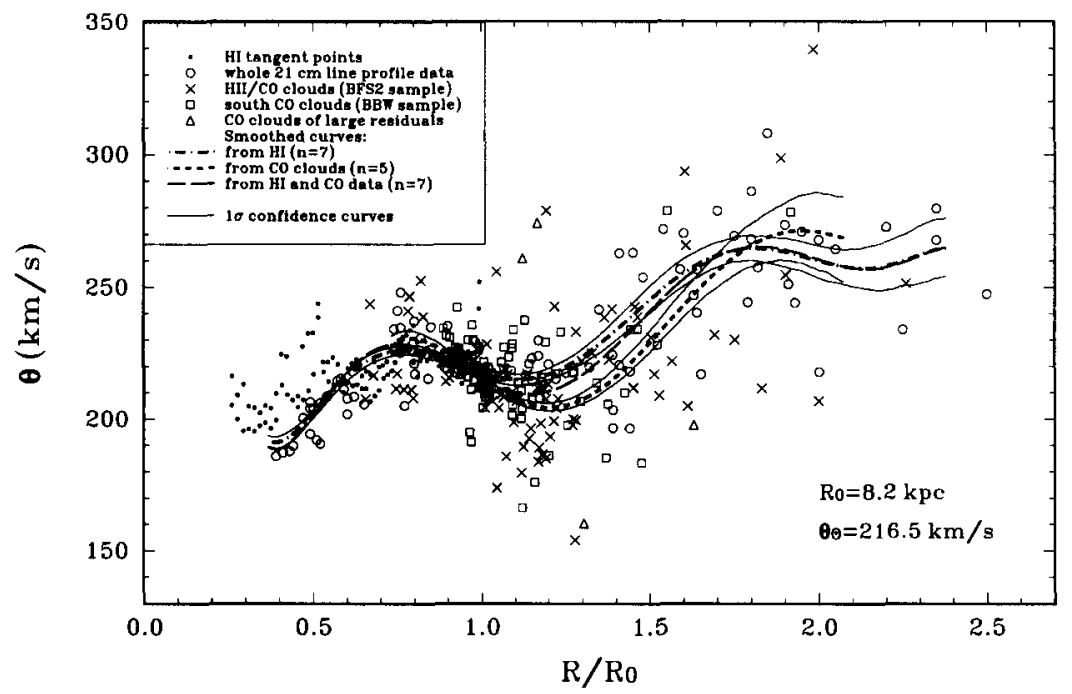

Figure 1. The neutral hydrogen and CO data on the Galaxy rotation and rotation models constructed in this paper.

"Acceptable" degrees $n$, for which polynomial $W_{n}(x)$ represents well all the reliable structure in the data, were determined in the manner suggested by Nikiforov (1999a,b). Objects with large residuals have been excluded.

\section{Results and discussion}

The obtained $R_{0}$ estimates are summarized in Table 1 . Column (4) gives the result derived by an analysis of the $V_{r}$ field of COCs without using $\mathrm{H}$ I data (Nikiforov 1999b). Column (5) gives the weighted average of values in columns (2)-(4). The average of all six $R_{0}$ values in the latter columns was taken as the final estimation from COC distances: $R_{0}=8.2 \pm 0.7 \mathrm{kpc}$ (here the scatter of results was added in quadrature). Values obtained via mere $W(\mathrm{CO}+\mathrm{H} \mathrm{I})$ fitting, as in NP94, are listed in the last column; in this case $R_{0}$ is underestimated.

Table 1. $\quad R_{0}$ from $\mathrm{CO}$ clouds with and without using $\mathrm{HI}$ data.

\begin{tabular}{cccccc} 
Sample & $\begin{array}{c}W(\mathrm{CO}) \\
\text { fitting }\end{array}$ & $\begin{array}{c}W / V_{r} \\
\text { fitting }\end{array}$ & $\begin{array}{c}V_{r} \\
\text { fitting }\end{array}$ & Average & $\begin{array}{c}W(\mathrm{CO}+\mathrm{H} 1) \\
\text { fitting }\end{array}$ \\
\hline BFS2 & $8.52_{-0.54}^{+0.57}$ & $7.59_{-0.46}^{+0.50}$ & $8.26_{-0.77}^{+0.82}$ & $8.03 \pm 0.57$ & $7.12_{-0.25}^{+0.31}$ \\
BFS2/BBW & $8.56_{-0.34}^{+0.36}$ & $7.92_{-0.34}^{+0.35}$ & $8.87_{-0.54}^{+0.57}$ & $\mathbf{8 . 3 4} \pm 0.39$ & $7.93_{-0.30}^{+0.33}$ \\
\hline Average & $\mathbf{8 . 5 5 \pm 0 . 4 2}$ & $\mathbf{7 . 8 1} \pm 0.40$ & $\mathbf{8 . 6 7 \pm 0 . 6 4}$ & $\mathbf{8 . 2 4} \pm \mathbf{0 . 4 6}$ & $7.48 \pm 0.30$
\end{tabular}

Adopting the value for $R_{0}$ found above we obtain values of $A R_{0}=141.0 \pm 4.0$ $\mathrm{kms}^{-1}, A=17.20 \pm 0.48 \mathrm{~km} \mathrm{~s}^{-1} \mathrm{kpc}^{-1}$ from all $\mathrm{HI}$ and CO data, the LSR 


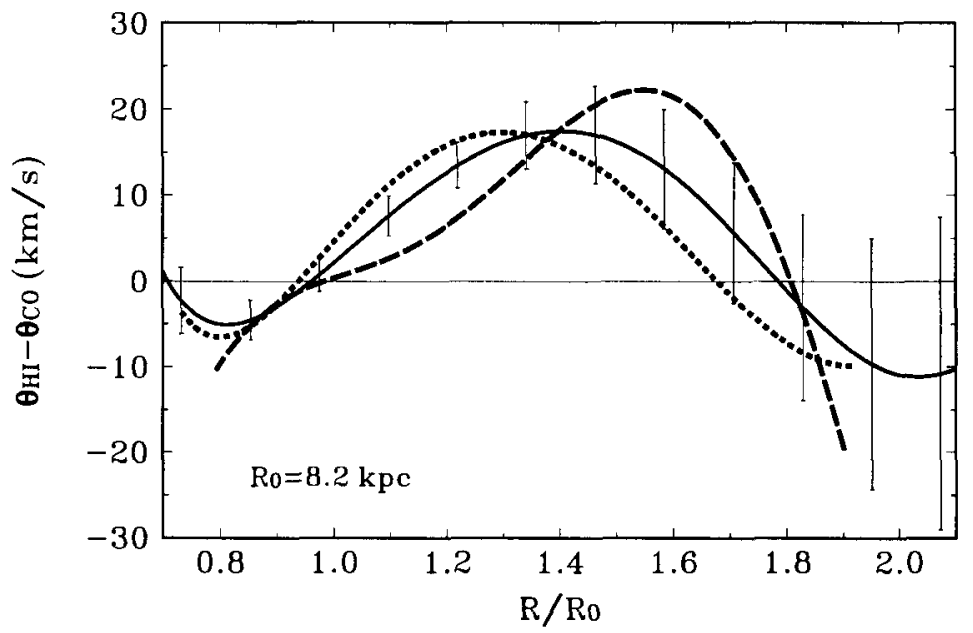

Figure 2. Differences between the smoothed rotation curve for $\mathrm{H} \mathrm{I}$ and the ones found for all $\mathrm{CO}$ clouds (solid line), clouds with $0^{\circ} \leq l<$ $180^{\circ}$ (short dashed line) and clouds with $180^{\circ} \leq l<360^{\circ}$ (long dashed line). Bars show the result of errors in smoothed values of $\theta_{\mathrm{H}_{\mathrm{I}}}$ and $\theta_{\mathrm{CO}}$.

motion relative to the COCs of $\Delta \theta_{\mathrm{LSR}}=2.6 \pm 1.3 \mathrm{~km} \mathrm{~s}^{-1}, \Pi_{\mathrm{LSR}}=-2.8 \pm 1.2$ $\mathrm{km} \mathrm{s}^{-1}$, Galactic gas's linear rotation velocity at the sun of $\theta_{0}=215 \pm 24 \mathrm{~km} \mathrm{~s}^{-1}$ and $\theta_{\mathrm{LSR}}=216.5 \pm 24 \mathrm{~km} \mathrm{~s}^{-1}$ (with the Kerr \& Lynden-Bell (1986) value of $\left.\omega_{0}=26.4 \pm 1.9 \mathrm{~km} \mathrm{~s}^{-1} \mathrm{kpc}^{-1}\right)$.

The $\mathrm{H}$ I and $\mathrm{CO}$ rotation curves and their 1- $\sigma$ confidence limits, found by Monte Carlo simulations, are shown in Figure 1. The linear rotation velocity $(\theta)$ of COCs is found to be, in average, $4.9 \pm 2.2 \mathrm{~km} \mathrm{~s}^{-1}$ less than the $\mathrm{H}$ I value. However the difference $\left\langle\theta_{\mathrm{HI}}-\theta_{\mathrm{CO}}\right\rangle$ turns out not to be constant (see Figure 2): it is significant at the $3-\sigma$ level only for $1.09_{+0.08}^{-0.04} \leq x \leq 1.46 \pm 0.02$.

Hence the suggested techniques account for only the net effect of the radiusaverage $\mathrm{H}$ I-CO difference on $R_{0}$. However with a modelling this difference in detail, the comparision with $\mathrm{H}$ I rotation becomes meaningless.

The smoothed rotation curve for $\mathrm{H}$ I is hardly affected by streaming motions because the whole $21-\mathrm{cm}$ line profile data represent all galactocentric azimuths, in contrast to COCs, and owing to a direct correction (Malahova \& Petrovskaya 1992). Therefore the $l$-dependent $\mathrm{HI}$-CO difference (cf. the north and southern CO-curves in Figure 2) may be considered as an effect of a spiral wave, namely the Perseus arm, on the COCs. This allows us to estimate directly the pitch 
angle of the arm as $22^{\circ} \pm 3^{\circ}$, which coincides with Blitz's (1983) result from H I kinematics for $x>1$ and supports the four-arm picture for the spiral structure of our Galaxy.

The drop in $\theta$, found by NP94, of $\sim 20 \mathrm{~km} \mathrm{~s}^{-1}$ at $0.75<x<1.15$ on average, is confirmed and traced from both $\mathrm{H} \mathrm{I}$ and $\mathrm{CO}$ data individually (Figure 1). This feature may be a "signature" of a dip in disk density (Nikiforov et al. 1999), which can be responsible for the existence of warps seen in the outer regions of the Galaxy. Both $\mathrm{H}$ I and $\mathrm{CO}$ data show a rise in $\theta$ at $1.1-1.2<x<1.7-1.8$.

Acknowledgments. This work is supported by the Russian Foundation for Basic Research (grant no. 96-02-19636) and by the State Integrated Research and Development Program "Astronomiya" of Russia (problem no. 1.2.3.2).

\section{References}

Blitz, L. 1983, in Surveys of the Southern Galaxy, W. B. Burton and F. P. Israel, Dordrecht: Reidel, 1983, 117

Brand, J. and Blitz, L. 1993, A\&A, 275, 67

Kerr, F. J. and Lynden-Bell, D. 1986, MNRAS, 221, 1023

Malahova, Yu. N. and Petrovskaya, I. V. 1992, A\&A Trans., 1, 221

Merrifield, M. R. 1992, AJ, 103, 1552

Nikiforov, I. I. 1999a, Astrofizika, 42, 399

Nikiforov, I. I. 1999b, Astronomy Reports, 43, 345

Nikiforov, I. I. 1999c, submitted to Kinematika i Fizika Nebesnykh Tel

Nikiforov, I. I. and Petrovskaya, I. V. 1994, Astronomy Reports, 38, 642

Nikiforov, I. I., Petrovskaya, I. V. and Ninković, S. 1999, these proceedings 